\title{
Parental acceptance of the utilization of silver diamine fluoride on their child's primary and permanent teeth
}

This article was published in the following Dove Press journal: Patient Preference and Adherence

\author{
Sara M Bagher' \\ Heba J Sabbagh' \\ Samer M AlJohani ${ }^{2}$ \\ Gahida Alharbi ${ }^{2}$ \\ Mariam Aldajani ${ }^{1}$ \\ Heba Elkhodary ${ }^{3,4}$ \\ 'Department of Pediatric Dentistry, King \\ Abdulaziz University, Jeddah, Saudi \\ Arabia; ${ }^{2}$ King Abdulaziz University, \\ Jeddah, Saudi Arabia; ${ }^{3}$ Department of \\ Pediatric Dentistry, Faculty of Dentistry, \\ King Abdulaziz University, Jeddah, Saudi \\ Arabia; ${ }^{4}$ Department of Pediatric \\ Dentistry, Faculty of Dental Medicine for \\ Girls, Al Azhar University, Cairo, Egypt
}

Correspondence: Sara M Bagher Department of Pediatric Dentistry, King Abdulaziz University, Jeddah, Saudi Arabia Tel +966547433525

Email sbagher@kau.edu.sa
Purpose: Silver diamine fluoride (SDF) is an effective caries control agent. The aim of our study was to investigate the parental acceptance of the utilization of SDF on their child's primary and permanent teeth and to determine the factors that influence their decisionmaking.

Patients and methods: This descriptive cross-sectional study included parents of healthy children aged 12 years and younger and currently receiving dental treatment. The interview questionnaire was conducted and pre-tested for face and content validity. The trained interviewing dentists showed colored pictures of primary and permanent teeth before and after receiving SDF treatment. The statistical significance was set at $P<0.05$.

Results: A total of 104 parents were included in the study. The mean parental rating of treatment acceptability of the staining associated with SDF was 3.9 \pm 1.95 . The plurality considered the staining caused by SDF treatment strongly not acceptable 46 (43.4\%). Parental acceptance of SDF treatment was significantly affected by the location and type of teeth. Parents showed significantly higher acceptance of SDF treatment on their child's primary compared to permanent teeth and posterior compared to anterior in both dentitions $(P<0.001)$. In addition, parents of children with a history of uncooperative behavior during previous dental treatment were significantly more accepting of SDF treatment regardless of the type and location of the teeth.

Conclusion: Parental acceptance of SDF increased for primary compared to permanent teeth, on anterior compared to posterior teeth in both dentitions and for uncooperative children.

Keywords: caries, permanent teeth, preference, primary teeth, silver diamine fluoride, staining

\section{Introduction}

Dental treatment of young pre-cooperative children can be challenging and often a pediatric dentist must employ more advanced behavior management techniques including protective stabilization and/or sedation or general anesthesia to be able to perform the required treatment and arrest the lesions' progression. ${ }^{1}$ Therefore, it is of great importance to identify an effective, low-cost method for treating caries lesions in children. Silver diamine fluoride (SDF) has been identified as a costeffective, easy, and simple topical cariostatic agent comprised of silver and fluoride, used in the management of caries and dentin hypersensitivity. ${ }^{2}$ It works by forming a squamous layer on the exposed dentin and partially plugging the dentinal 
tubules. ${ }^{3}$ The silver compounds arrest caries by interfering with the ability of the pathogens to form a biofilm on the SDF-treated surface. Also, it encourages remineralization, with the development of fluorapatite from the first hydroxyapatite crystals. ${ }^{3}$

The use of SDF was cleared by the Food and Drug Administration in the United States of America in August 2014 as a desensitizing agent. ${ }^{4}$ The University of California, San Francisco School of Dentistry (UCSF) released a standardized protocol, rationale, indications, and consent form for the use of SDF in dentistry in $2016 .{ }^{4}$ At UCSF, SDF is indicated for patients who cannot tolerate standard care for medical or psychological reasons and for patients with extreme caries risk and cannot be treated conventionally. ${ }^{4}$

The use of $38 \%$ SDF off-label by licensed professionals has proven to be safe and effective in controlling dental caries in young children in several large clinical trials. $^{2,5-7}$ Twice per year application was shown to be more effective in caries control compared to once a year until the tooth is restored or exfoliates. ${ }^{6,7}$

In 2016, Nelson et al, reported that SDF is rapidly being adopted among the US pediatric dentistry residency programs as a caries control agent in children. Parental acceptance, cost, and obtaining the product were the most perceived barriers to the use of SDF for pediatric patients. ${ }^{8}$

Few published studies evaluated the parental acceptance of the utilization of SDF on their children's teeth. Although color change associated with its application was the main barrier, ${ }^{9-11}$ many of them will prefer SDF treatment over general anesthesia and further invasive treatment. ${ }^{11}$ A recent review by $\mathrm{Hu}$ et al, in 2018 reported that most of the parents were satisfied with the cosmetic outcome of SDF. Children also found it comfortable and relatively pain-free procedure. ${ }^{12}$ While most of the parents rejected such treatment for their children teeth. ${ }^{13}$ To our knowledge, there are no previously published data in the literature that compared the parental acceptance of the utilization of SDF on their children's anterior and posterior teeth in Jeddah, Saudi Arabia. Therefore, the aim of our study was to investigate the parental acceptance of the utilization of SDF on their child's primary and permanent teeth and to determine the factors that influence their decision-making the most at King Abdulaziz University Faculty of Dentistry in Jeddah, Saudi Arabia. We hypothesized that parents will be more accepting of the utilization of SDF on their child's primary compared to permanent teeth, and more in posterior compared to anterior teeth. We also hypothesized that staining associated with the application of SDF will be the most common barrier to parental acceptance of the treatment.

\section{Material and methods}

This descriptive cross-sectional study was carried out at King Abdulaziz University Faculty of Dentistry in Jeddah, Saudi Arabia (KAUFD) pediatric dental clinics in Jeddah, Saudi Arabia for dental treatment between December 2017 and February 2018. The study was approved by the Research Ethics Committee of the Faculty of Dentistry at King Abdulaziz University (069-0.16). Inclusion criteria include; parents of healthy children aged 12 years and younger attending pediatric dental clinics and agree to participate in the study. Sample size calculation was carried out using open-epi online program with $80 \%$ power. The expected number of patients attending the pediatric clinics in a two-month period was estimated retrospectively to be 418 children, giving a sample size of 104 parents. An Arabic letter explaining the aim of the study along with a consent form was signed by the participating parent before taking part in the study.

\section{Questionnaire validation}

The interview questionnaire was conducted after a literature review, and it included information about the participating parents and their participating child. It was pre-tested for both face and content validity. To test the content validity, up to five faculty members at KAUFD were given the same survey individually and asked to rate each question using a 5 -point Likert scale. For the face validity, a total of 20 questionnaires were distributed to a representative sample. They were asked to offer their opinions regarding each question and whether they had any trouble understanding or answering any of the questions. The examiners were trained at a formal meeting that provided detailed explanations regarding the questionnaire and the questionnaire coding manual and rubrics.

If the participating parents had more than one child attending KAUFD for dental treatment, they were asked to answer about their youngest child. The interview was performed by two trained general dentists. The first section of the questionnaire included sociodemographic data. The family's economic state was divided into three groups based on the family's total household income in Saudi Riyals per month; the groups included low, moderate, and high. In the second section, parents were asked about their child's behavior during previous dental visits, 
history of pain, and about the most important factor in selecting the type of treatment for their child.

The interviewing dentists showed high-quality colored pictures of cases of primary and permanent teeth diagnosed with caries before and after treatment using SDF. They also explained the steps of application, cost, advantages, and disadvantages of using SDF briefly. Then, the participating parent was asked to rate the treatment acceptability regarding the staining and rank their acceptance as $1=$ strongly acceptable, $2=$ acceptable, $3=$ neutral, $4=$ not acceptable, and $5=$ strongly not acceptable. Later, they were asked if they would consider using SDF for their child's anterior and posterior primary and permanent teeth. The factor(s) that influenced their decisions the most were recorded.

\section{Statistical analysis}

The collected data were tabulated and statistically analyzed using Windows SPSS Software (version 12.1). Chisquare test was used for nominal data and independent sample $t$-test for means comparison. Ordinal regression analysis was carried out to estimate covariate adjusted associations with the dependent variable (parental acceptance to staining associated with SDF treatment). The factors and covariates included child's age, gender, the key sociodemographic predictors (parental gender, education level, and family income), child cooperation, and history of pain. The statistical significance was set at $P<0.05$.

\section{Results}

Out of the 180 parents invited to participate in the research, 104 (43 fathers and 61 mothers) completed the questionnaire giving a response rate of $57.8 \%$. The children ages ranged between 2 and 12 years with a mean \pm SD age of $7.27 \pm 2.35$ and most of them were 8 years and older 47 (45.19\%). The sociodemographic data of the participating subjects are represented in Table 1.

When asked about the most important treatment goal, $77(74.0 \%)$ parents reported that "no pain" was the most important factor in selecting the type of treatment, compared to $27(26 \%)$ reporting esthetics to be the most important. Child's gender, parents' gender, parents' educational level, and history of pain did not show significant effects on the parents' most important treatment goal $(P>0.05)$. Although not significant, parents of children with history of uncooperative behavior during previous dental treatment and parents of high-income tend to prefer esthetics compared to parents of uncooperative children
Table I Sociodemographic characteristics of participating subjects $(\mathrm{N}=104)$

\begin{tabular}{|l|l|}
\hline Variable & $\mathrm{N}(\%)$ \\
\hline Child age (Years) & \\
$5-4$ & $13(12.5)$ \\
$8-12$ & $44(42.3)$ \\
Male & $47(45.19)$ \\
Female & $59(56.7)$ \\
\hline Parents & $45(43.3)$ \\
Mother & $61(58.7)$ \\
Father & $43(41.3)$ \\
\hline Parental education & $63(60.6)$ \\
$\leq$ High school & $4 \mathrm{I}(39.4)$ \\
\hline University and higher education & $68(65.4)$ \\
\hline Family income & $23(22.1)$ \\
Low & $13(12.5)$ \\
\hline Moderate & $31(29.8)$ \\
Nooph & $40(38.4)$ \\
\hline Cooperation during pervious dental treatment & \\
\hline History of pain & $(27.0)$ \\
\hline
\end{tabular}

and low income $(P=0.084$, OR:3.19\% and $95 \%$ CI: $0.856-$ 11.88, $P=0.053$, OR:0.32\% and 95\% CI: $0.1-1.01$, respectively) (Table 2).

When the parental acceptance to the utilization of SDF was questioned, almost half of the parents reported that it is strongly not acceptable $46(43.4 \%)$ due to the staining caused by its application. On the other hand, $16(15.1 \%)$ of them were neutral and only $2(1.9 \%)$ found the staining acceptable. The mean parental rating of acceptability to the staining associated with SDF treatment was $3.9 \pm 1.95$. When regression analysis was carried out, parental acceptance of the staining associated with SDF treatment was not affected by the child's gender, parent's gender, parental education level, family income, and history of pain $(P>0.05)$. 
Table 2 Distribution of the most important treatment goal among participating parents $(\mathrm{N}=104)$

\begin{tabular}{|c|c|c|c|}
\hline Variable & Cosmetic N (\%) & Pain N (\%) & $P$-value, $O R$ and $95 \%(\mathrm{Cl})$ \\
\hline \multicolumn{4}{|l|}{ Child gender } \\
\hline Male & $15(25.4)$ & $44(74.6)$ & $0.909,1.054(0.429-2.585)$ \\
\hline Female & II (24.4) & $34(75.6)$ & \\
\hline \multicolumn{4}{|l|}{ Parents } \\
\hline Mother & $13(21.3)$ & $48(78.7)$ & $0.301,0.61(0.256-1.528)$ \\
\hline Father & $13(30.2)$ & $30(69.8)$ & \\
\hline \multicolumn{4}{|l|}{ Parents' education } \\
\hline$\leq$ High school & $13(20.6)$ & $50(79.4)$ & $0.203,0.56(0.228-1.374)$ \\
\hline$\geq$ University and higher education & $13(31.7)$ & $28(68.3)$ & \\
\hline \multicolumn{4}{|l|}{ Family income } \\
\hline Low* & $5(16.1)$ & $26(83.9)$ & $0.828,0.87(0.235-3.186)$ \\
\hline Moderate & $6(18.2)$ & $27(81.8)$ & $0.053,0.32(0.1-1.01)$ \\
\hline High & $15(37.5)$ & $25(62.5)$ & \\
\hline \multicolumn{4}{|c|}{ Cooperation during pervious dental treatment } \\
\hline Cooperative* & $22(32.4)$ & $46(67.6)$ & $0.084,3.19(0.856-11.88)$ \\
\hline Uncooperative & $3(13.0)$ & $20(87.0)$ & \\
\hline Do not know & I (7.7) & $12(84.6)$ & \\
\hline \multicolumn{4}{|l|}{ History of pain } \\
\hline Yes & $17(22.4)$ & $59(77.6)$ & $0.45,0.608(0.233-1.587)$ \\
\hline No & $9(35.7)$ & $19(64.3)$ & \\
\hline
\end{tabular}

Note: *Reference-comparison group.

Parental acceptance of SDF treatment was significantly affected by the location and type of teeth. Parents showed significantly higher acceptance of SDF in their child's primary anterior and posterior compared to permanent anterior and posterior teeth $(P<0.001)$ (Table 3$)$. Also, they showed significantly higher acceptance of SDF treatment in their child's posterior compared to anterior teeth in both primary and permanent dentitions $(P<0.001)$ (Table 3$)$. In addition, the child's cooperation significantly affected parental acceptance. Parents of children with a history of uncooperative behavior during previous dental treatment were significantly more accepting of SDF treatment compared to parents of cooperative children regardless of the type and location of the teeth (Table 4).

\section{Discussion}

To our knowledge, this is the first study to investigate the parental acceptance of the utilization of SDF on their child's primary and permanent teeth and to examine the factors that influence their decision the most at KAUFD in Jeddah, Saudi Arabia. In all of the previously published studies, only the parental acceptance of the utilization of SDF on their child's teeth was evaluated and compared between anterior and posterior teeth. ${ }^{1,9,13}$ In our study, parental acceptance of the utilization of SDF on their child's teeth was evaluated and compared between primary and permanent teeth along with anterior and posterior teeth.

Significantly more parents accepted the staining associated with SDF treatment on their child's primary compared to permanent teeth, and also on posterior compared to anterior teeth in our study. This agrees with the findings reported by recent studies in which most of the parents accepted the staining on posterior more than on anterior teeth. ${ }^{9,11}$ This can be because parents understood that their child will lose the primary teeth eventually and that the staining will be much less visible on posterior teeth.

In a recent study reported by Gordon in 2018, parental acceptance of the utilization of SDF on their children was higher among parents of uncooperative children or children who require more advanced behavior management. ${ }^{9}$ This agrees with our findings, in which the acceptance of SDF treatment among parents of children with a history of uncooperative behavior during previous dental treatment significantly increased when compared with parents of cooperative children regardless of the location and type of teeth. This showed that the parents in our study are willing to 


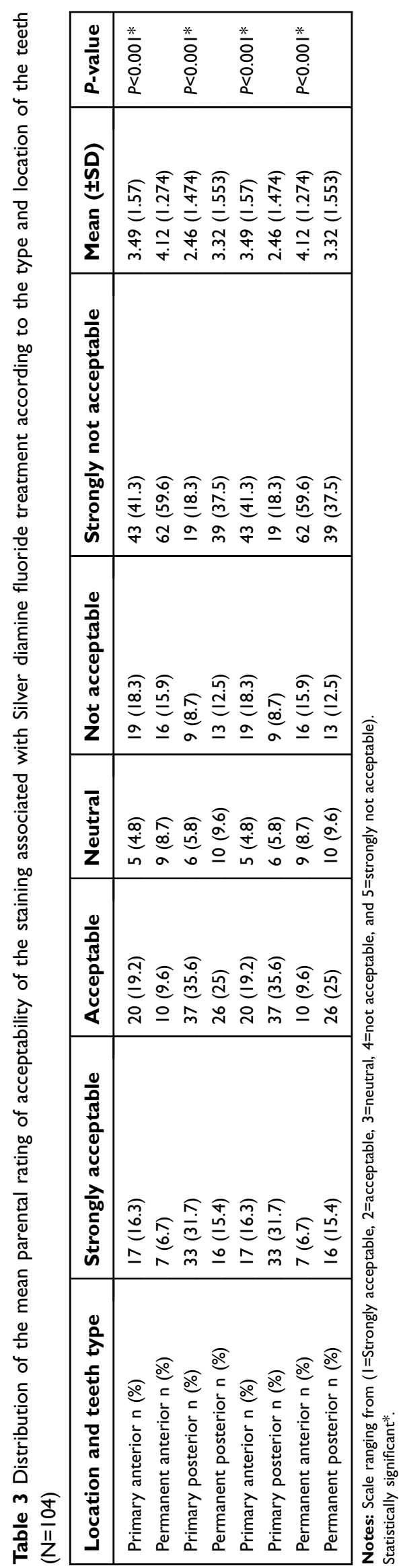


Table 4 The mean parental rating of acceptability of the staining associated with Silver diamine fluoride treatment according to child cooperation, teeth location, and type $(\mathrm{N}=104)$

\begin{tabular}{|c|c|c|c|}
\hline Parental acceptance & Cooperative & Mean (士SD) & $P$-value \\
\hline \multirow[t]{2}{*}{ Anterior primary } & Yes & $3.76(1.5)$ & $P=0.00 I^{*}$ \\
\hline & No & $2.52(1.47)$ & \\
\hline \multirow[t]{2}{*}{ Posterior primary } & Yes & $2.78(1.57)$ & $P<0.00 I^{*}$ \\
\hline & No & $1.48(0.67)$ & \\
\hline \multirow[t]{2}{*}{ Anterior permanent } & Yes & $4.28(1.26)$ & $P=0.027^{*}$ \\
\hline & No & $3.57(1.47)$ & \\
\hline \multirow[t]{2}{*}{ Posterior permanent } & Yes & 3.71 (1.53) & $P<0.00$ I* \\
\hline & No & $2.30(1.36)$ & \\
\hline
\end{tabular}

Notes: Scale ranging from (I=Strongly acceptable, 2=acceptable, 3=neutral, 4=not acceptable, and 5=strongly not acceptable).

Statistically significant *

compromise their child's esthetic appearance if it means the child can receive treatment without the need for more advanced behavior management techniques. The same conclusion was reported by Clemens et al, and Crystal et al ${ }^{10,11}$.

In both of the previously reported studies, ${ }^{9,11}$ the questionnaire was administrated in both English and Spanish languages either electronically or on paper. In our study, two trained general dentists interviewed the parents and explained all the advantages and disadvantages of the utilization of SDF. All the parents were shown the same pictures to ensure consistency. On the other hand, in a recent study by Clemens et al, in 2017, the parents' satisfaction was assessed immediately after the application of SDF and most of the parents agreed or strongly agreed on the ease of SDF application with an acceptance of the discoloration associated with SDF treatment. ${ }^{10}$

There were some limitations in our study; first of all, the parents were asked about their child's behavior during previous dental treatment without having that behavior evaluated by a trained dentist. Moreover, the study recruited a sample from KAUFD which is a governmental hospital in which the treatment is provided free of charge. Therefore, our findings may not be generalized over the population. Also, parents were only shown pictures of before and after treatment cases. None of the parents who participated had a child who had received SDF treatment. A lack of such exposure might have affected their decision and preference regarding such treatment. Therefore, studies on larger samples including parents of children who had received SDF treatment are required to provide more conclusive and generalized findings.

When the children's discomfort associated with SDF treatment was compared to resin infiltration in treating initial carious lesions, significantly less discomfort and higher acceptability were reported with SDF treatment. ${ }^{14}$ In addition, in our study, only the parents' acceptance was evaluated based on photos of treated cases and none of the children's opinion or preference was recorded.

Our findings highlight the importance of following the American Academy of Pediatric Dentistry Guidelines ${ }^{15}$ in evaluating child and parental circumstances before introducing and providing SDF treatment, which also emphasizes the need of utilizing clear and effective informed consent with proper photos and description of the advantages and disadvantages before providing such treatment. Given our results, SDF is a simple, easy, cost-effective, and well-accepted treatment for uncooperative patients regardless of the type and location of the teeth. Pediatric dentists should consider SDF as a highly recommended treatment option in uncooperative patients for caries management especially for primary and posterior teeth.

\section{Conclusion}

Parents' main goal of treating their children's teeth was pain elimination. Parental acceptance of SDF increased for primary and posterior teeth compared to permanent and anterior teeth and for children with a history of uncooperative behavior during previous dental treatment.

\section{Author contributions}

All authors contributed to data analysis, drafting or revising the article, gave final approval of the version to be published, and agree to be accountable for all aspects of the work.

\section{Disclosure}

The authors report no conflicts of interest in this work. 


\section{References}

1. American Academy on Pediatric Dentistry. Behavior guidance for the pediatric dental patient. Pediatr Dent. 2016;40:254-267.

2. Chu CH, Mei ML, Lo EC. Use of fluorides in dental caries management. Gen Dent. 2010;58:37-43.

3. Mei ML, Ito L, Cao Y, et al. Inhibitory effect of silver diamine fluoride on dentine demineralisation and collagen degradation. $J$ Dent. 2013;41:809-817. doi:10.1016/j.jdent.2013.06.009

4. Horst JA, Ellenikiotis H, Milgrom PM. UCSF protocol for caries arrest using silver diamine fluoride: rationale, indications and consent. $J$ Calif Dent Assoc. 2016;44:16-28.

5. Gotjamanos T. Pulp response in primary teeth with deep residual caries treated with silver fluoride and glass ionomer cement ('atraumatic' technique). Aust Dent J. 1996;41:328-334.

6. Yee R, Holmgren C, Mulder J, et al. Efficacy of silver diamine fluoride for arresting caries treatment. J Dent Res. 2009;88:644-647. doi: $10.1177 / 0022034509338671$

7. Zhi QH, Lo EC, Lin HC. Randomized clinical trial on effectiveness of silver diamine fluoride and glass ionomer in arresting dentine caries in preschool children. $J$ Dent. 2012;40:962-967. doi:10.1016/j. jdent.2012.08.002

8. Nelson T, Scott JM, Crystal YO, et al. Silver diamine fluoride in pediatric dentistry training programs: survey of graduate program directors. Pediatr Dent. 2016;38:212-217.
9. Gordon NB. Silver diamine fluoride staining is acceptable for posterior primary teeth and is preferred over advanced pharmacologic behavior management by many parents. Evid Based Dent Pract. 2018;18:1894-1897.

10. Clemens J, Gold J, Chaffin J. Effect and acceptance of silver diamine fluoride treatment on dental caries in primary teeth. $J$ Public Health Dent. 2017;7:1-6.

11. Crystal YO, Janal MN, Hamilton DS, et al. Parental perceptions and acceptance of silver diamine fluoride staining. $J$ Am Dent Assoc. 2017;148:510-518. doi:10.1016/j.adaj.2017.03.013

12. Hu S, Meyer B, Duggal M. A silver renaissance in dentistry. Eur Arch Paediatr Dent. 2018;19:221-227. doi:10.1007/s40368-0180363-7

13. Alshammari AF, Almuqrin AA, Aldakhil AM, Alshammari BH, Lopez JNJ. Parental perceptions and acceptance of silver diamine fluoride treatment in Kingdom of Saudi Arabia. Inter $J$ of Heal Sci. 2019;13:25-29.

14. Mattos-Silveira J, Floriano I, Ferreira FR, et al. Children's discomfort may vary among different treatments for initial approximal caries lesions: preliminary findings of a randomized controlled clinical trial. Int J Paediatr Dent. 2015;25:300-304. doi:10.1111/ipd.12134

15. AAPD. Policy on the use of silver diamine fluoride for pediatric dental patients. Pediatr Dent. 2017b;39:51-53.

\section{Publish your work in this journal}

Patient Preference and Adherence is an international, peer-reviewed open access journal that focusing on the growing importance of patient preference and adherence throughout the therapeutic continuum. Patient satisfaction, acceptability, quality of life, compliance, persistence and their role in developing new therapeutic modalities and compounds to optimize clinical outcomes for existing disease states are major areas of interest for the journal. This journal has been accepted for indexing on PubMed Central. The manuscript management system is completely online and includes a very quick and fair peer-review system, which is all easy to use. Visit http:// www.dovepress.com/testimonials.php to read real quotes from published authors. 